2018-11-26

\title{
Development of a low powered wireless iot sensor network based on mbse
}

Taha, J

http://hdl.handle.net/10026.1/13684

10.1109/SysEng.2018.8544420

4th IEEE International Symposium on Systems Engineering, ISSE 2018 - Proceedings

All content in PEARL is protected by copyright law. Author manuscripts are made available in accordance with publisher policies. Please cite only the published version using the details provided on the item record or document. In the absence of an open licence (e.g. Creative Commons), permissions for further reuse of content should be sought from the publisher or author. 


\section{Development of a Low Powered Wireless IoT Sensor Network based on MBSE}

\author{
Jihad Taha \\ Department of Applied Sciences \\ and Mechatronics \\ Munich University of Applied \\ Science \\ Munich Germany \\ Jihad.Taha@hm.edu
}

\author{
Vahid Salehi \\ Department of Applied Sciences \\ and Mechatronics \\ Munich University of Applied \\ Science \\ Munich Germany \\ Salehi-d@hm.edu
}

\author{
Frank Abraham \\ Associate Professor Mechanical \\ \& Marine Engineering \\ University of Plymouth \\ Plymouth England \\ frank.abraham@plymouth.ac.uk
}

\begin{abstract}
Nowadays, the trend in System Engineering (SE) is shifting more and more into the domains of Wireless Sensor Networks (WSN) and Internet of Things (IoT) and will reach new height in demand. The complexity of those systems which are fundamentally different from those of the last decades in their own areas and are furthermore growing. To fulfill all requirements in those fields, a wider range of functionalities and components are needed, especially for IoT. In the interdisciplinary development, many conflicts occur. Thus, the complexity of the product demands a change of the development process itself. Most approaches lack in aspects of transparency, traceability and continuous development in general. Therefore, a neutral systematic approach is needed, which model-based systems engineering (MBSE) could provide. This paper applied MBSE in the domain of IoT in WSN development. Although MBSE emerged from system engineering/development, it essentially allows any developer in any discipline to understand relations between different system components in the central system model. The stage of this research is not fully developed yet but a high potential is expected. This holistic central system model is described by MBSE with tool, modelling language and method. The most promising modelling language for an interdisciplinary work in a real industrial content is SysML, which will be applied throughout the modelling tool following the RFLP principle of the V-model. These endeavours will then represent our result to show a more transparent continuous traceable solution in comparison with the existing application.
\end{abstract}

Keywords-MBSE, SysML, IoT, WSN

\section{INTRODUCTION}

The need to measure and monitor different physical or environmental conditions, such as temperature, humidity, pressure or container status, on a dynamic, infrastructure independent level is highly required, not only in an industrial perspective but also many other fields [1]. While in industrial aspects the global competition resulting into less development and production time, the quality must still be meet [2]. A system is needed to monitor and probe many points. According to this description, a Wireless Sensor Network (WSN) have the potential to fulfill these needs of probing and monitoring in not only industrial application but also to be applied in many more domains [3] [4].

Depending on the application location and measurement targets, implementing different sensor type into specified WSN designs is inevitable. Typically, a WSN contains many sensor nodes. Those sensor nodes are equipped with sensors to sample the environment, a computing device to process the data, a power supply and a transmitter for a wireless communication. Nevertheless, the nodes are physically constrained and must work after deployment independently of any external inputs. The main objective of all nodes of a WSN is to collect information of interest and then transmit it to the data-sink or base station [5]. The traditional approach for mechatronic systems implementations and design are not sufficient for sensors in a WSN. Similar to the challenges by changing from 2D design to parametric 3D modelling, the constraints and fundamental differentness must be overworked and newly set [6].

Following the requirements for low complexity with low energy consumption, a proper balance must be found [7]. A promising method to research and development WSN is the MBSE with the SysML, which can lead to potentials in low energy devices, computational efficiency and other domains which are now untouched. The MBSE describes three domains, which are visually described in Fig.1.

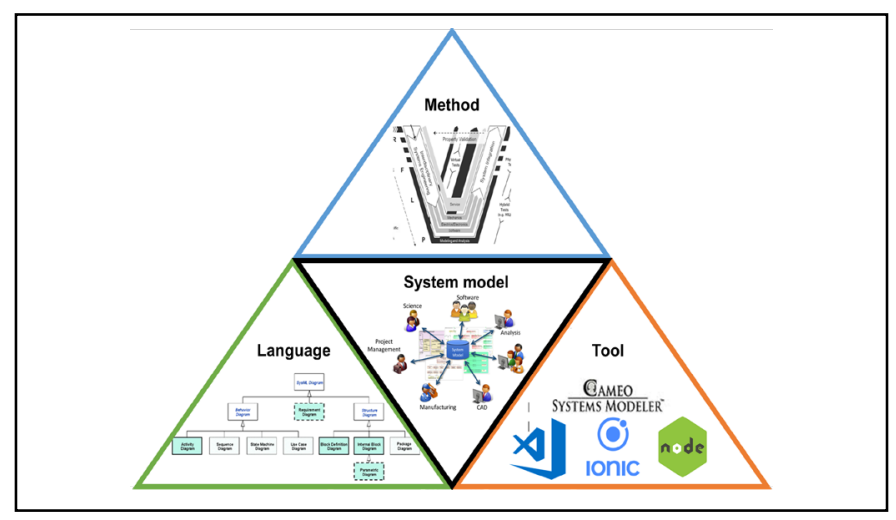

Fig. 1. MBSE Pyramid [8] 
The Fig.1 shows the domains of the method, language, and tool. All together, they describe a subject in a seamless, systematic and neutral way. Thus, the correct application of the MBSE with SysML as language will yield significantly abstractive benefit in all interdisciplinary areas and will support even follow-up projects [9] [10]. The Cameo Systems Modeler could be one of the tools to implement the system modelling. It is also necessary to be mentioned that MBSE emerged from systems engineering, although similar leaned approaches exists, which describe overlapping domains [11].

This paper will discuss and elaborate how MBSE with SysML as Modelling language will affect the need of transparency, traceability and continuous development in an interdisciplinary environment, with all related and applied subjects and domains shown in the Fig.2. Firstly, all the subjectrelated terms in WSN and MBSE with SysML will be explained through a literature survey and summarized state of the art in section two. The following section will outline the resulting challenges and research gaps. The fourth section will show the results of the application of MBSE with SysML in the development and implementation of a low powered wireless IoT sensor network. The first part of the fourth section will explain the used tool, modeling language and method in terms of MBSE, followed by a description of the used method, then an in-depth description of the implementation description of the resulting modeling language in the used tool. Lastly, the final section will summarize the achieved advantages in this subject.

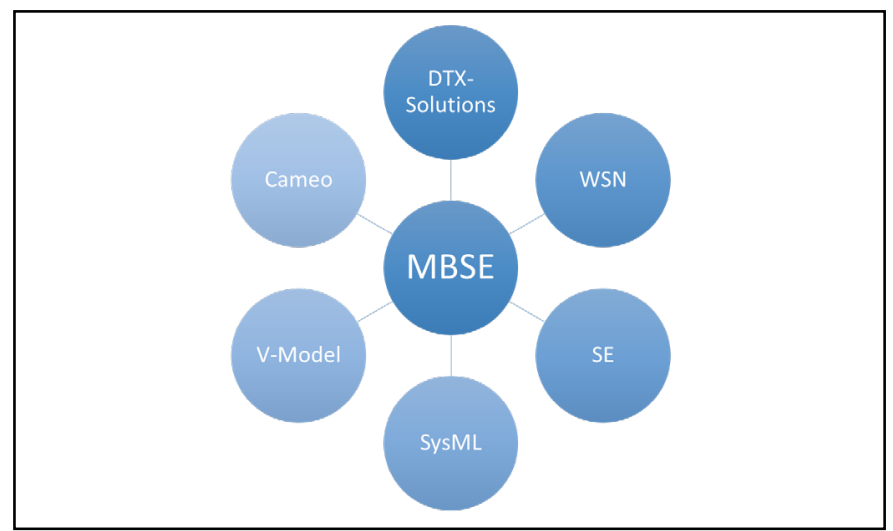

Fig. 2. Overview of all Systems

\section{LITERATURE SURVEY AND STATE OF THE ART}

This section defines WSN and MBSE, as well as their subterms, and summarize their applications. This will help to understand all following sections.

\section{A. Wireless Sensor Network}

The WSN is described as a "self-configured and infrastructureless wireless networks to monitor physical or environmental conditions, such as temperature, sound, vibration, pressure, motion or pollutants and to cooperatively pass their data through the network to a main location or sink where the data can be observed and analyzed." [1] by M.A. Matin and M.M. Islam. This establishes discussion topics in this research field.
To begin with, the sensors of a WSN are called nodes. Those nodes will sense the environment and allow environmental values to be captured. These values can be transferred if needed and processed afterwards. Four main basic components must be available to accomplish this. These components are shown in Fig. 3 [3] [12].

- The sensing unit, also known as sensor, will convert the analog environmental data into digital data. It is the main interface to the environment.

- The processing unit, which contains also the storage, processes the not only the sensor data but also manage the logical code and the communication with other nodes.

- To allow a communication a transceiver unit is needed, which receive and send message, thus connect the node to WSN.

- The last basic unit is the power unit. This can be a passive power supplier like a battery or an active one, like a landline.

Depending on the application of the node and WSN, many more unit can be implemented to fulfill any needed function. A key feature of such nodes is to work at minimized power consumption. The transmitter is usually the largest power consumer. Therefore, it is recommended to use a power control unit, like turning the node off [3] [12].

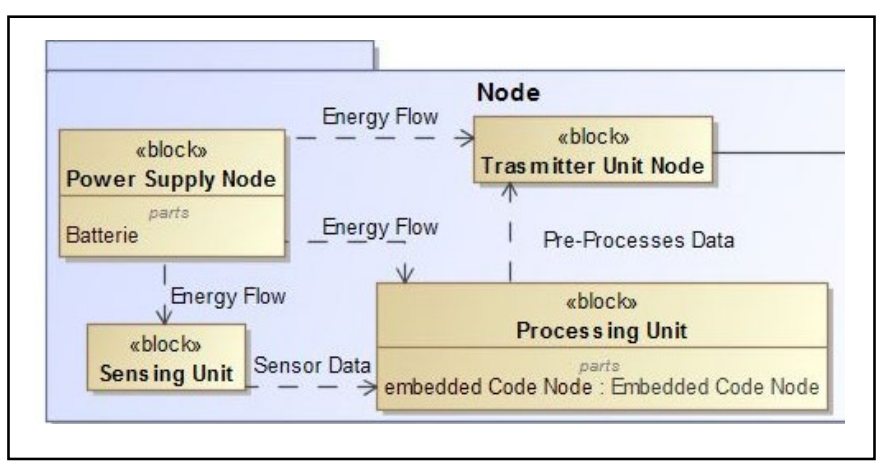

Fig. 3. Overview Node buildup in Logical components from Cameo

The topology of the WSN defines the structure of the network itself and thus the used protocol structures. Following are the most common topologies for WSN.

- The "star network" communication topology is one of the most common topologies for WSN. Here multiple remote nodes use a single base station to communicate. This base station can send and/or receive messages and processes them for further use. Between each node, communication is not allowed. The simplicity, low latency and the ability for low power operation of the node are advantages of this network. The disadvantage is that the single centralized base station must be within the range of all nodes and in case of a failure, the entire network will break down. Compared to other networks this is the least robust one [1]. 
- A "mesh network", also known as Peer to Peer (P2P) network allows the communication between each node within a certain range. This network has the potential to cover larger areas than the previous one. If any communication target node is not within range, a socalled "multi-hop communications" can be done. This allows nodes to send messages to another node, which will forward the message to the target node. This can happen over multiple nodes until the target node is reached. The main advantages of this network topology are redundancy and scalability. Due to the lack of centralized communication a simple node failure will result in near no effect on the network, as long as a remote node still can communicate to any other node in its range and so on. The range of the network is only limited to the number of nodes. By adding more nodes to the network a scalable grow is possible. The high power consumption of each node is the largest disadvantage of this network type. To allow a multi-hop communication, each node must be ready to operate at any time. The latency will also grow with the number of nodes for the communication to reach the target node as well as increase the power consumption [1].

- It is also possible to have any combination of the network types, depending on the use case scenario. This hybrid provides a robust and versatile communication network and will maintain the ability of minimum power consumptions for each nodes. In this network topology, there are two types of nodes: the lowest power consumption which is not able to forward messages like described in the star network, and those with the ability to forward messages like described in the mesh network. Thus the nodes with the ability to forward message enable the multi-hop function and allow to carry the message from the low power nodes to the target node or base [11].

\section{B. $M B S E$}

To understand MBSE, not only a definition is needed but also an insight into the most used methodologies. Two definitions of MBSE are from NDIA Systems Engineering Division [13] and from INCOSE SE Vision 2020 [14].

NDIA defines MBSE as: "Model-Based Engineering (MBE): An approach to engineering that uses models as an integral part of the technical baseline that includes the requirements, analysis, design, implementation, and verification of a capability, system, and/or product throughout the acquisition life cycle." [13] while INCOSE SE Vision 2020 MBSE definition is: "Model-based systems engineering (MBSE) is the formalized application of modeling to support system requirements, design, analysis, verification and validation activities beginning in the conceptual design phase and continuing throughout development and later life cycle phases." [14].

In Short, the application of MBSE is defined as the interaction of modelling language, method, and tool [15]. J. A. Estefan summarized the most widely used application of MBSE. The most striking are followed [16]:
- The first interesting methodology application is from Vitech CEO and Chief Methodologies James. E. Long. Here the approach is spread out on multiple day courses, while the Methodology itself is based on four main system engineering activities. Functional/Behavior analysis, Source Requirements Analysis, Architecture Synthesis, Design Validation, and Verification. It is stated that to set up and manage this approach, a good System Definition Language in MBSE is needed.

- IBM Rational Unified Process for Systems Engineering for Model-Driven Systems Development focus on the need of systems engineering projects. The discipline and best practices of Rational Unified Process was applied to its new development, specifically for software development in areas of systems specification, analysis, design, and development.

\section{SysML}

The graphical modelling language SysML is based on UML and is targeted to help by specifying, analysing, designing, and verifying complex systems in a neutral and unequivocally way. Hardware, software, information, personnel, procedures, and facilities can be described [17].

Lenny Delligatti describes one of the most suited definition: "SysML is one of several graphical modelling languages. The key word is language. SysML is a language - a medium for communicating ideas from one person to another. It has a grammar and a vocabulary just like any of the natural languages we speak (e.g., Hindi, Japanese, English). SysML is the language "spoken" by MBSE practitioners when they create system models to visualize and communicate ideas about their systems' designs to other stakeholders" [18].

SysML is used to support the development processes of mechatronic systems. The language was released 2007 by the Object Management Group (OMG), an international technology standards consortium [17]. While UML is nearly entirely adopted to SysML, many new concepts have been added and omitted [19]. Weilkiens describes the most important extensions of SysML as [20]:

- Adding the requirement diagram

- Supporting the data type ISO AP-233 to enable data exchange between different tools

- Renaming and upgrading diagram types

To describe and connect the requirements with the structure and behaviour, SysML will support and ease the endeavour. Thus the two areas of application are as documentary language and development supporting tool.

\section{MBSE/SysML and WSN/IoT}

Within the scope of the literary survey, two publications are most striking in the combination of domains of MBSE and WSN/IoT.

The first one is the IEEE paper "Reuse in Model-Based Systems Engineering" from U. Shani and H. Broodney [9]. They describe the long-term advantages of MBSE in a project and 
follow-up project. Strictly used on project a harvesting for reusable component for new projects is possible. The example is described by identifying a component in a modelling tool and then used in the new project by adding new contexts and adapted it to the new use-case, while possible using another tool as before. Here the focus is on the conflict within the inter-tool communication by this procedure. This is called as "tools interoperability". Some solutions are described. Most striking possible solutions for this tool information transfer problem within MBSE is the Open Services for Lifecycle Collaboration (OSLC). OSLC link the data with web services. These model managing servers provide a web resources similar solution in which the engineers can browse similar as any other web resources. Each model needs a server to manage them, instead a client application on the engineers side, which is the disadvantage.

The second paper is "Enabling Design of Agile Security in the IOT with MBSE" from B. L. Papke [21]. He describes how the threat to IoT devices is agile and evolving over time. And in response to this threat, system security features must become agile and evolving part of the development. He describes first how it is possible to response to this agile threat. Here he is referring to Dove, R. "Sustainable Agile Security Enabled by Systems Engineering Architecture" which proposed an approach for sustainable agile security. This should be possible by an agile systems architecture and concept of operations. Four fundamental elements are required:

- "An open agile architecture pattern that includes a catalog of drag-and-drop encapsulated modules and the module pools in which they belong." [21].

- "A passive infrastructure defined by a set of agile design principles that address aspects of system reusability, reconfigurability and scalability and response requirements addressing the proactive and reactive domains to achieve system agility." [21].

- "An active infrastructure that defines the parties responsible for module mix and evolution, module readiness, system assembly and infrastructure evolution." [21].

- "The resulting reconfigurable and scalable system configurations responsible for providing the business/operational system functionality with resilience.” [21].

Considering now the domains SysML and WSN alone, multiple endeavours are available. Here the focus of the literature is set on different targets. Following are some striking application attempts.

Y. B. Maissa in "A SysML profile for wireless sensor networks modeling" describe how the requirements for SysML as modelling language is needed to be applied in the area of WSN [22]. The result is a SysML profile model for a wireless sensor networks. Other applications of SysML with WSN from A. Hammad in "An Approach Combining SysML and Modelica for Modelling and Validate Wireless Sensor Networks" illustrates an approach to design and to verify the energy consumption property of a WSN using the Model Driven Engineering standards [23].

In the named literature SysML is applied as modelling langue in combination of WSN, while the focus is set on specific areas of interest [22] [23] [24] [25]. Even application of MBSE with WSN are focused not on the development of WSN, but also specific point, like Agile Security or reusability [21] [9]. A throughout continues application of MBSE with SysML for an overall WSN development did not occur.

\section{DEVELOPMENT GAPS}

Emerged from the literature survey and implementation of MBSE in a WSN/IoT, the question if any appropriate approaches in terms of MBSE in WSN/IoT development have been adapted is still open. Even U. Shani and H. Broodney [9] and B. L. Papke [21] use MBSE in only specific areas of interest and SysML for modelling WSN are used on specific applications and without any development method [22] [23] [24] [25]. Throughout the literature survey, a fully implementation of MBSE into continues development of an IoT device and WSN is nearly untouched. Even applications of WSN describe only the usage of such system and not the systematic development. Many developments and applications of WSN have been already proceeded and the most interesting ones are as listed here:

- S. Ferdoush and X. Li in the development of a WSN for Environmental Monitoring system [26].

- H. Kumbhar in the development of a WSN test environment in combination of Xbee on an Arduino [27].

- P. Wuttidittachotti and S. Kilaso in the development of a WSN Weather and Disaster Alarm Systems [28].

- H. Hermawan in the Healthcare monitoring application in ubiquitous sensor network [29].

This shows that like described in section II that no fully attempt to apply MBSE with SysML to develop in a continuous and transparent way, from the first step of the V-Model to the application, is done in this way.

Therefore, this paper describes the endeavor of the development of a low powered wireless IoT sensor network based on MBSE, although MBSE is focused on system engineering, [16] it will set the example and profile how to implement, describe and apply the SysML model appropriately of the V-Model RFLP within such progress. The implementation itself contains many development challenges, especially in the aspect of a low powered wireless IoT device. 


\section{SYSTEM DESIGN AND IMPLEMENTATION}

To accomplish a successful implantation with MBSE, each section, language, method and tools, must be predefined. In this case, the modeling language is SysML, described by OMG [17], which will be used in the System Modeler "Cameo" throughout the entire implementation to support the developer. Additionally, the commonly used method V-Model (VDI2206) is applied here, with the Requirement/Function/Logic/Physical (RFLP) view. Therefore, a continuous systematic development can take place. Following the visual description of MBSE in Fig. 1, Fig. 4 shows an overview of the applied component.

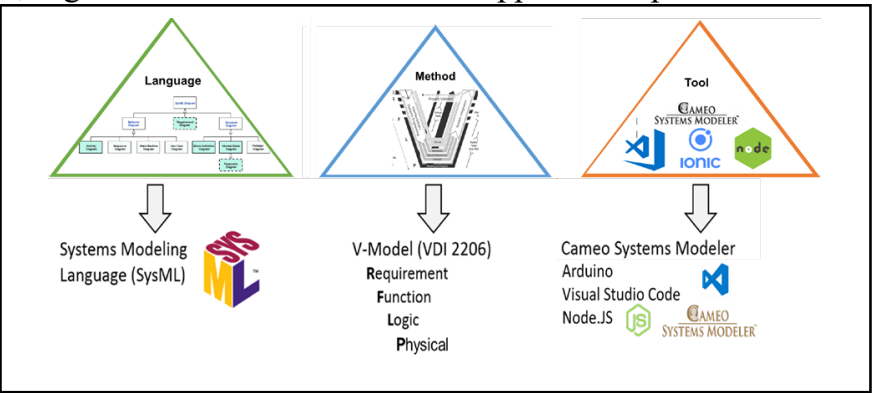

Fig. 4. MBSE applied component [8]

\section{A. RFLP of the V-Model}

The V-Model is a commonly used method for developing mechatronic products but can also be adapted for specialized cases [30]. This method is built by two branches shown in Fig. 5. The left side is the 'Systems Engineering (SE)' phase and the right side is the 'System Integration phase'. The SE phase should guide the development with the RFLP steps. Each step helps to describe the "to be developed product" from the rough overview down to the smallest detail [31].

Requirement: The first step is the definition of requirements for the system and subsystems. Salehi and Burseg elaborate different kind of requirements to allow a more depth description and elaborations. The two most important one are User and Functional requirements [32]. These requirements can also separate in quantitative and qualitative requirements. While little text can be used to describe a requirements of a system, a splitting and refiment of these requirements can lead to a clearer understanding of what is needed. The usage of words "shall" and "must" defines requirements which the system should and which must contain.

Functions: The second step is the functions which satisfy the previously defined requirements. Depending on the complexity of the requirements, any given function of the system must be included to fulfill the requirements.

Logical: The third step is to define the logical components to be able to describe the system. Here parts, APIs and similar components are shown to set how the system should be able to perform the functions. Commonly behavior diagrams, like an activity or system flow diagrams, are used in this phase.

Physical: The last step is to set the real physical components which concentrates on the solution approach. Each function must be fulfilled with a physical component which leads to the full product.

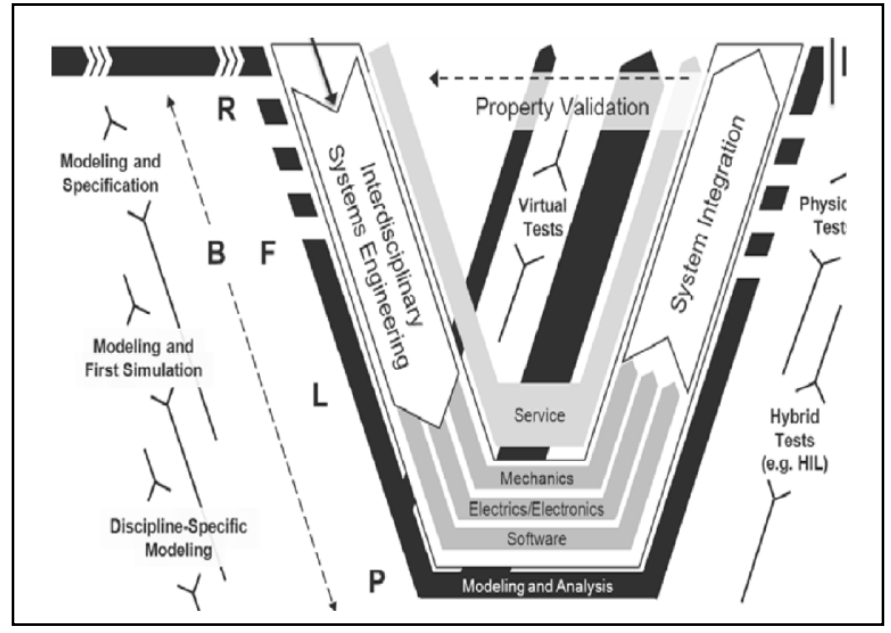

Fig. 5. V-Model [33]

\section{B. SysML and Cameo}

SysML provides many diagrams and descriptions for systems. Depending on the system, certain diagrams are useful. SysML does not have a modeling guideline yet, especially in case of applicable tools like the Cameo Systems Modeler. As a result, users have to propose their own approach. In this usecase, only the important diagram will be mentioned coherent to the RFLP. These diagrams are categorized into structural views and behavioral views. These structural views are in short the definition of components and connectors, in which variations are defined with internal block diagrams. The block definition diagram is used to specify the types of components and connectors [34]. The behavioural views are definitions of activities, behaviour and related actions. Here the used blocks express a specified resulting activities and actions. The aim is to describe the behaviour of component, systems, configurations and similar subject in term of interaction, data, dataflow and control [34]. The implementation process of this project is divided into 4 parts, which are strictly following the V-Model method with so-called RFLP steps as shown in Fig.5.

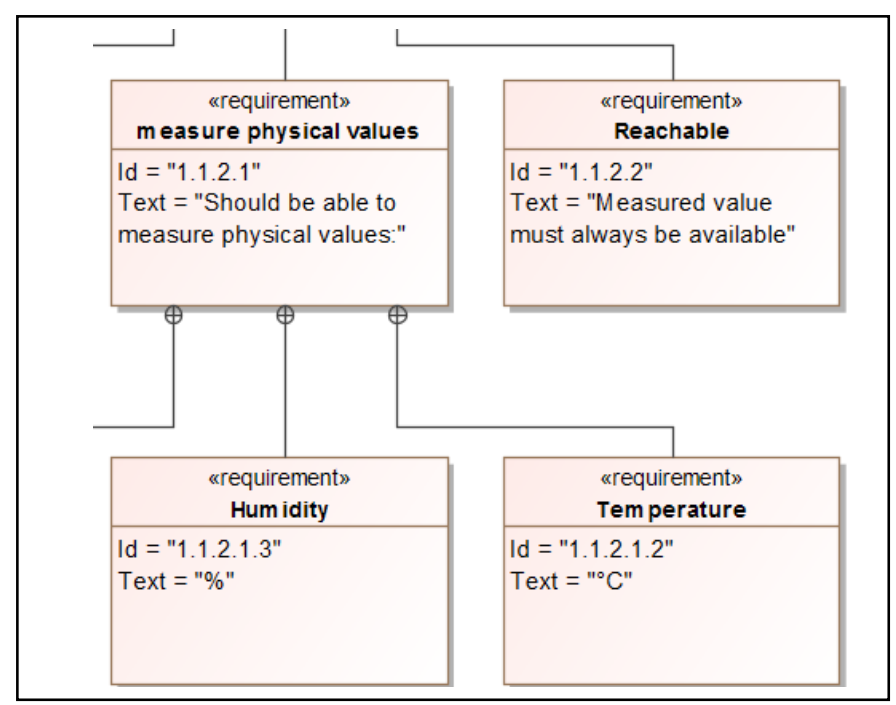

Fig. 6. Requirement Diagram 
To ease the explanation of a low powered wireless IoT sensor network throughout the RFLP, only the sensor example will be used and explained. The first and most important part is the description of the requirements, which includes all kinds of mechanical, electrical and software requirements. Here all functional and none-functional requirements are listed by category as depicted in Fig.6. The requirements are phrased by specific rules with describing the necessity by using "shall" and "must". The phrasing of each element describes which part of the sub-system is responsible of the request.

The next diagram of the system model is a function diagram. Here each function is directly linked to the corresponding requirement which fulfills or effects. All functions are shown in the function hierarchy. Every function has been split up into subfunctions, which describe more specific basic functions or constrain the parent function. For instance, if the function is defined as "Able to Measure environment" it is constrained by the sub-function "must be Wireless". These sub-function are linked with "satisfy" to the requirement from which they originally arose. Fig. 7 depicts the sub-functions of "Measure environment" which is split into a single function to read the distance, humidity, and the temperature. The fulfilled requirements are on top linked with 'satisfy' relation.

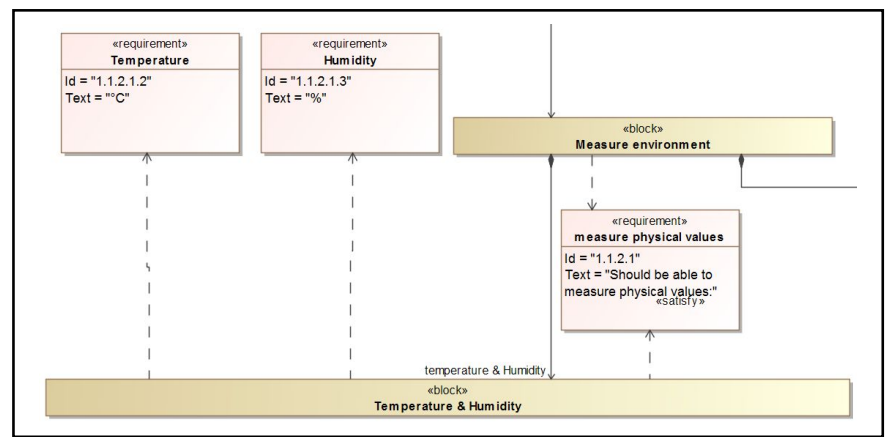

Fig. 7. Function Diagram

The next diagram is the logical diagram, which is coupled to the activity diagram. This is done so, to explain the logical function in a holistically continuous way. Most functions have to be described together, as they depend and influence each other. Again, here the entire linkage to the requirement can be followed back. The "Sensor-Functions" fulfills all requirements. The logical description of the system is seen in the following Fig. 8 and is describing the logical view of all components. These components are selected to fulfill the functions and so also the requirements. The components show the energy, data, and material flow. It should be noted here that the material flow is not required.

An activity diagram support explanation of certain complex functionality in a logical view. Here all activities of a system are described with all possible reaction of the named system. It includes all steps of a use-case from launching the system to the stage when fulfilled its purpose. A detailed step by step installation will help the developer greatly to explain how such system works, how functions are used and how it satisfies the requirements. In our implementation case, the activity diagram explained how the sensor works. The sensor initiates by including the batteries. This will start the embedded code. After each code step has been executed, the code checks if any error has occurred. If so, the sensor will shut down until the pre-set interval has passed, before trying again. In case of no error, the sensor scans the environment and sends the data to the data-sink.

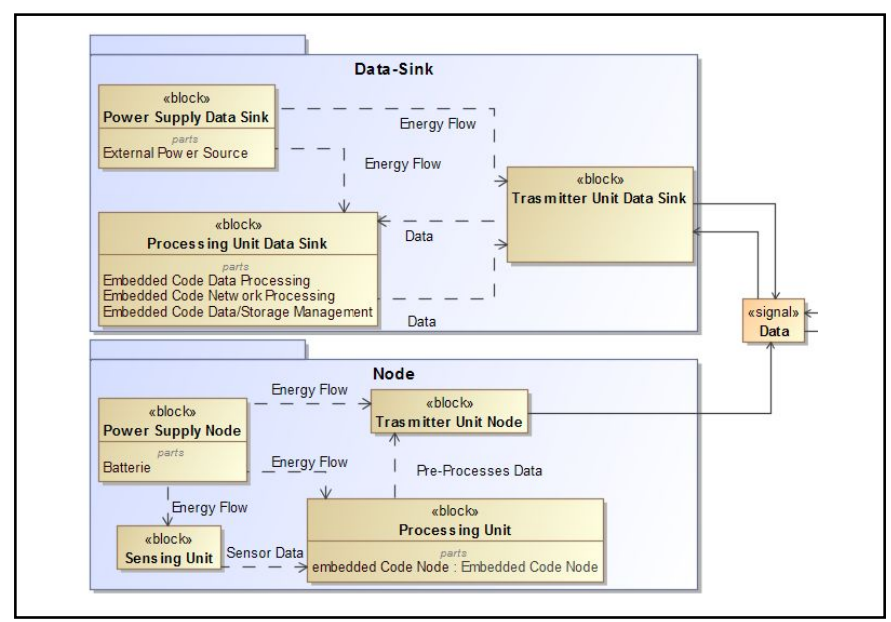

Fig. 8. Logical Diagram

The physical diagram in Cameo will conclude the entirety RFLP of the V-Model. Here the selected physical components are linked through all diagrams to the requirements. It is visible how the linked physical component are grouped and which requirements affects them directly.

To ease the description, the lowest level of the requirement and functionality can be linked to the physical components. In Fig. 9, the example of the sensor is described how the components are connected together.

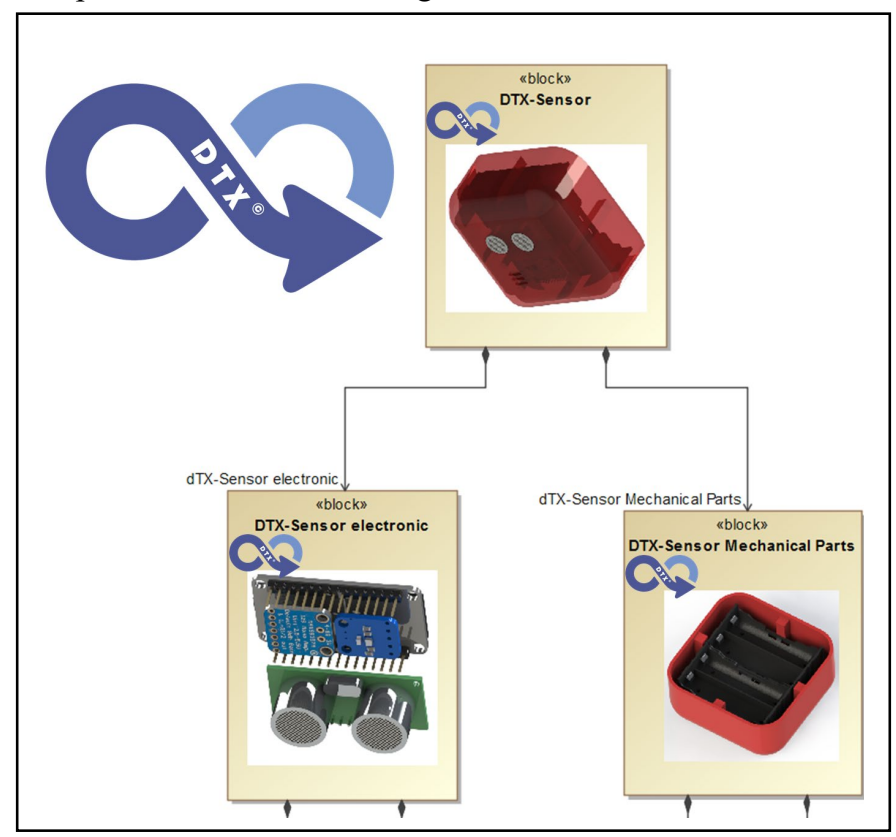

Fig. 9. Physical Diagram

All of these diagrams help to describe a product or a system and keep everything simple despite interdisciplinary descriptions. Additionally, one more step to explain and to describe the product could be helpful. With a matrix, the 
interaction between each created diagram element, within a diagram or inter-diagrams can be displayed. In the parametric developments and design of mechatronic systems, many matrices, like Parameter Structure Matrix or associative structure matrix, are applied so support and visualize dependencies. [35]

The example is given in Fig. 10 that functions-requirements interaction is created and displayed. This matrix shows how the functions satisfy the requirement. In this case, it could help the developer to keep the track of the unsatisfied requirements.

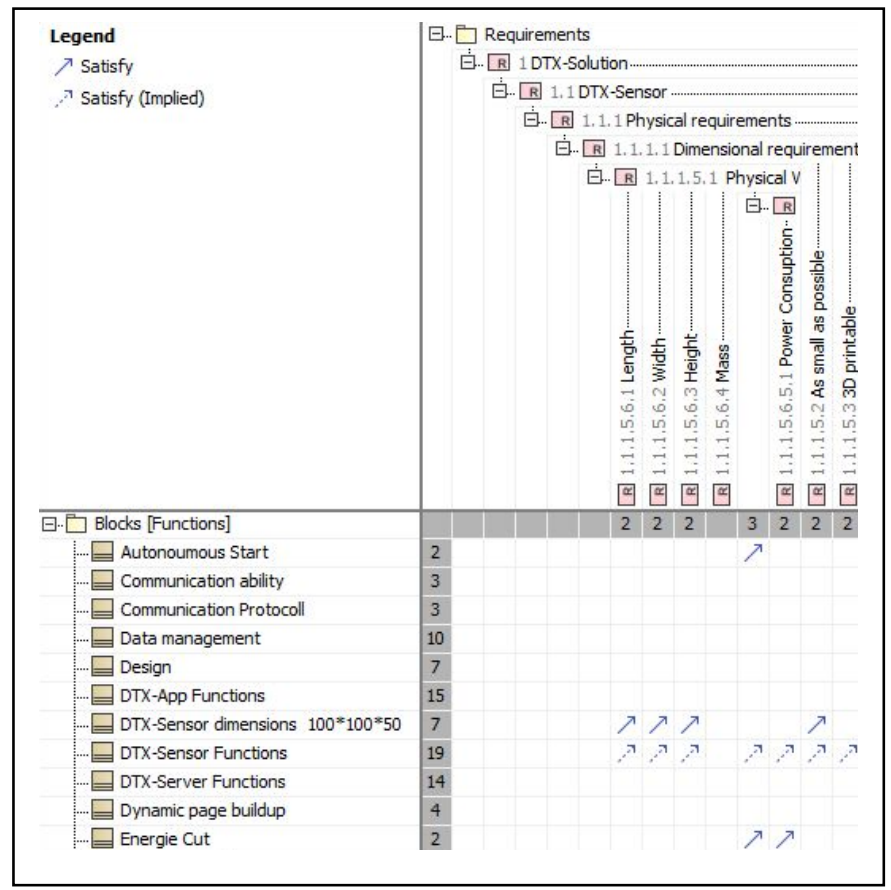

Fig. 10. Functions-Requirements Matrix

The function-requirements matrix is an inter-diagram matrix, but the importance of none inter-diagram matrices is not negligible. One of those matrices is the requirementsrequirements matrix. Here the dependencies, effects, inter action and more other relations can be displayed. So in case of prioritizing of requirements, the effect can be traced back to all other linked requirements. This concludes the SysML implantation part.

\section{DTX-Solution}

The targeted development Solution is called DTX-Solution (Do Things eXcellent). The DTX-Solution contains three sub products: the DTX-Server, the DTX-App, and the DTX-Sensor.

- The DTX-Server functions as a back-end system. It will manage all connections and received data. This system operated completely autonomous and is based on Node.JS. Minor error will be detected and corrected within its programmed abilities.

- The DTX-App is an Android mobile app and displays all sensor data. Here the app contains the functionality to configure the sensors depending on their application area.

- The DTX-Sensors are low powered wireless IoT sensors. According to Akyildiz's description of how those sensor nodes are built and called [3], the four components of these nodes are: 1) the US-100 ultra-sonic and the SI7021 as the sensing units, 2) the Node MCU V2 as the processing unit which contains the embedded code 3 ) the ESP8266 as the transmitter unit and 4) the 4 Li-Ion batteries as the power supply.

The entirety of MBSE, SysML and RFLP approach have been applied of the whole DTX-Solution, but for simplicity reasons only described on the DTX-Sensor. According to the definition of WSN by M.A. Matin and M.M Islam [1], the DTXSensor is a node within a star-topologies. Every DTX-Sensornode is set with an interval to sense the environment, sends the data to the data-sink and then turns itself off to save a tremendous amount of power. The data-sink and the gateway that allows accessing the WSN and sensor-data are the DTXServer, while the DTX-App is an outside consumer, which accesses the data through the gateway.

\section{CONCLUSION}

The advantages of combining SysML with Cameo System Modeller alone are in this project significant, but through further applying MBSE and its three domains, tools, Language and Methods, the results did lead to more precise outcome.

A clear and exact description of the system with help of various diagrams, did lead to the benefits to understand compley interacting system within a short time similar described in [36]. SysML helps the project to clarify different subjects and can be used as discussion foundation in an interdisciplinary team. All key information are described in a way, which is understandable regardless of domain, own specialization or general subject. All sub-systems and components of the product are displayed and so it is easier to find solutions challenges or implement improvements. SysML is able to support a centralized file directory. Any file, instruction, survey, 3D-models and component information can be included directly in the system. This can be used to reduce time consumption of data management. Many users have second thoughts about its efficiency since MBSE and SysML are quite new procedures. Their benefits and potentials are not yet visible without actual utilization. Optimization is needed and will change this situation in the future. Moreover, the increasing popularity of MBSE will lead to a better acceptance of SysML.

Throughout this project, the usage of the systems modeler provided the advantages such as long-term time saving, misunderstanding prevention and less learning period due to the unambiguous description of each element in the system.

As described by U. Shani and H. Broodney, any model developed in MBSE can be reused in not only follow-up project but also in new projects [9].

Future follow-up projects and researches will focus on MBSE with SysML to not only consolidate the benefits and 
advantages but also to explain how they can be optimized [10] [9].

\section{REFERENCES}

[1] M. Matin and M. Islam, "Overview of WSN," 2012.

[2] V. Salehi and C. McMahon, "Methodological integration of parametric associative CAS systems in product lifecyle management (PLM) enviroment," in International Design Engineering Technical Conferences and Computers and Information in Engineering Conferences, San Diego, California, USA, 2009.

[3] I. F. Akyildiz, Y. S. W. Su and E. Cayirci., "Wireless sensor networks: A survey. Computer Networks," 2002.

[4] V. Salehi and C. McMahon, "Development of an evaluation framework for implementation of parametric associative methods in an industrial context," in Computer Aided Design and application, 2011.

[5] K. Akkaya and M. Younis, "A survey on routing protocols for wireless sensor networks," 2005.

[6] V. Salehi and C. McMahon, "Development and Application of an Integrated Approach for Parametric Associative CAD Design in an Industrial Context," in Computer-Aided Design \& Applications, 2011.

[7] C. Buratti, A. Conti, D. Dardari and R. Verdone, "An Overview onWireless Sensor Networks Technology and Evolution," 2009.

[8] V. Salehi, "Model Based Systems Engineering (MBSE) as a holistic Approach and Enabler for Autonomous Driving Systems," Hochschule für Angewandte Wissenschaften München, 2018.

[9] U. Shani and H. Broodney, "Reuse in Model-Based Systems Engineering," 2015.

[10] NoMagic, Why do MBSE?, 2012.

[11] V. Salehi and M. Chris, "Identification of Factors During the Introduction and Implementation of PLM Methods and Systems in an Industrial Context," Germany Munich, 2016.

[12] J. S. Wilson, Sensor Technology Handbook, Elsevier Inc, 2005.

[13] NDIA, "Final Report, Model-Based Engineering Subcommittee," 2011.

[14] INCOSE-TP2004-004-02, "INCOSE SE Vision 2020," 2007.

[15] J. Martin, "Systems engineering guidebook: A process for developing systems and products," 1997.

[16] J. Estefan, "Survey of Model-Based Systems Engineering (MBSE) Methodologies," 2007.

[17] O. M. Group, "About the Object Management Group," [Online]. Available: http://www.omg.org/gettingstarted/gettingstartedindex.htm. [Accessed 2904 2018].

[18] L. Delligatti, SysML distilled: A brief guide to the systems modeling language, Pearson Technology Group , 2014.

[19] D. Dori, Model-Based Systems Engineering with OPM and SysML, New York: 10.1007/978-1-4939-3295-5, 2016.
[20] T. Weilkiens and R. Soley, Systems Engineering mit SysML/UML, dpunkt.verlag, 2014.

[21] B. Papke, "Enabling Design of Agile Security in the IOT with MBSE," No Magic Inc., Texas, U.S., 2017.

[22] Y. B. Maissa und S. Mouline, ,A SysML profile for wireless sensor networks modeling, " in IEEE, 2010.

[23] A. Hammad, H. Mountassir und S. Chouali, „An Approach Combining SysML and Modelica for Modelling and Validate Wireless Sensor Networks, “" Montpellier, France, 2013.

[24] N. Ammar, H. Chaieb und R. Bouallegue, ,From Modeling with SysML to simulation with Contiki Cooja Simulator of Wireless Sensor Network, " in International Conference on Advanced Information Networking and Applications Workshops, 2016.

[25] S. Berrani, A. Hammad und H. Mountassir, „Mapping SysML to Modelica to Validate Wireless Sensor Networks Non-Functional Requirements," 2013.

[26] S. Ferdoush and X. Li, "Wireless Sensor Network System Design using Raspberry Pi and Arduino for Environmental Monitoring Applications," 2014.

[27] H. Kumbhar, "Sensor Network using Xbee on Arduino Platform," 2016.

[28] P. Wuttidittachotti, "A Wireless Sensor Network for Weather and Disaster Alarm Systems," 2011.

[29] H. Kemis, N. Bruce, W. Ping, T. Antonio, L. B. Gook and H. J. Lee, Healthcare Monitoring Application in Ubiquitous Sensor Network: Design and Implementation based on Pulse Sensor with Arduino, Taipei, Taiwan: IEEE, 2013.

[30] V. Salehi and S. Wang, "Using point cloud technology for process simulation in the context of digital factory based on a systems engineering integrated approach," in 21st International Conference on Engineering Design (ICED 17) Vol 3: Product,, Vancouver, Canada, 2017.

[31] M. Eigner, D. Roubanov and R. Zafirov, Modellbasierte virtuelle Produktentwicklung, Springer Vieweg Verlag.

[32] V. Salehi and L. Burseg, "System Driven Product Development (SDPD) by Means of Development of a Mechatronic Systems in an Industrial Context," Springer, 2015.

[33] M. Eigner, T. Gilz and R. Zafirov, "Proposal for functional product description as part of a PLM solution in interdisciplinary product development," in INTERNATIONAL DESIGN CONFERENCE DESIGN 2012, Dubrovnik - Croatia,, 2012.

[34] F. Oquendo, J. Leite and T. Batista, "Software Architecture in Action," Springer International Publishing, 2016.

[35] V. Salehi und C. McMahon, ,Development of a generic integrated approach for parametric associative CAD systems, " in International conference on engineering design, ICED 09, USA, Standford, 2009.

[36] V. Salehi, J. Taha and F. Groß, "Implementation of Systems Modeling Laguage (SysML) in Consideration of Consens Apprach," Internationa Design Conference - DESIGN 2018, 2018. 